\title{
WEAKLY STARLIKE MEROMORPHIC UNIVALENT FUNCTIONS
}

\author{
BY \\ RICHARD J. LIBERA AND ALBERT E. LIVINGSTON
}

\begin{abstract}
A weakly starlike meromorphic univalent function is one of the form $f(z)=-\rho z g(z)[(z-\rho)(1-\rho z)]^{-1}$ for $0<\rho<1$ and $g(z)$ a meromorphic starlike function. The behavior of ccefficients and growth of this class of functions and of a subset are studied.
\end{abstract}

1. Introduction. Let $f(z)$ be meromorphic in the open unit disk defined by $|z|<1$ and hereafter called $\Delta$ with a simple pole at $\rho, 0<|\rho|<1$, and otherwise regular in $\Delta . f(z)$ is in $\Lambda(\rho)$ if and only if there is a number $\rho_{1}$, $|\rho|<\rho_{1}<1$, such that

$$
\operatorname{Re}\left\{\frac{z f^{\prime}(z)}{f(z)}\right\}<0
$$

and

$$
\frac{1}{2 \pi} \int_{0}^{2 \pi} \operatorname{Re}\left\{\frac{z f^{\prime}(z)}{f(z)}\right\} d \theta=-1
$$

for $\rho_{1}<|z|<1$ with $z=r e^{i \theta}$. On the other hand, $f(z)$ is in $\Lambda_{1}(\rho)$ if and only if $f(z)$ is regualr in $\bar{\Delta}$, the closure of $\Delta$, except again for a simple pole at $\rho$ and (1.1) and (1.2) are satisfied on $\partial \Delta$, the latter being the boundary of $\Delta$. Clearly $\Lambda_{1}(\rho)$ is a subset of $\Lambda(\rho)$.

It is no restriction on the geometric conditions given in (1.1) and (1.2) to assume that $f(z)$ in $\Lambda(\rho)$ be normalized so that $f(0)=1$ and $\rho$ be real; hence we shall hereafter make these assumptions. Also, it is clear that functions in the class $\Lambda(\rho)$ are univalent.

Conditions (1.1) and (1.2) taken together require that the origin be omitted by every function in $\Lambda(\rho)$. Meromorphic functions with a normalization similar to the above have been studied by Ladegast in an interesting paper which apparently has been overlooked [5]. Furthermore, the normalization taken for $\Lambda(\rho)$ can be viewed as an analog of the Montel normalization for regular univalent functions [6].

Received by the editors July 27, 1973 and, in revised form, February 13, 1974.

AMS (MOS) subject classifications (1970). Primary 30A24, 30A32, 30A34, 30A40. 
If $f(z)$ is in $\Lambda_{1}(\rho)$, then the function

$$
g(z)=(z-\rho)(1-\rho z) f(z) /-\rho z
$$

is meromorphic in $\bar{\Delta}$ with a pole of residue 1 at the origin. Furthermore on $\partial \Delta$,

$$
\begin{aligned}
\operatorname{Re}\left\{\frac{z g^{\prime}(z)}{g(z)}\right\} & =\operatorname{Re}\left\{\left(\frac{\rho}{z-\rho}\right)-\left(\overline{\frac{\rho}{z-\rho}}\right)+\frac{z f^{\prime}(z)}{f(z)}\right\} \\
& =\operatorname{Re}\left\{\frac{z f^{\prime}(z)}{f(z)}\right\}<0 .
\end{aligned}
$$

Consequently, $g(z)$ is in $\Sigma^{*}$ the class of meromorphic, normalized, starlike univalent functions ([1], [7]).

However, if we choose $f(z)$ in $\Lambda(\rho)$ we can likewise show that $f(z)$ has a representation like (1.3). For if we let $f_{r}(z)=f(r z)$, then $f_{r}(z)$ is in $\Lambda_{1}(\rho / r)$ for $\rho_{1}<r \leqslant 1$ and by the argument given above we infer the existence of a function in $\Sigma^{*}$ such that

$$
f_{r}(z)=\frac{-\rho z / r}{(z-\rho / r)(1-\rho z / r)} g_{r}(z) .
$$

$\Sigma^{*}$ is normal and compact [2], therefore we can find a sequence of increasing real numbers $\left\{r_{n}\right\}$ converging to 1 such that $\left\{g_{r_{n}}(z)\right\}$ converges to a function $g(z)$ in $\Sigma^{*}$ in compacta. Letting $n \rightarrow \infty$, we get the representation

$$
f(z)=-\rho z g(z) /(z-\rho)(1-\rho z)
$$

for every function $f(z)$ in $\Lambda(\rho)$.

(1.4) gives a particularly useful representation for studying the properties of $\Lambda(\rho)$; for this reason we define the following classes of functions. $f(z)$ is in $\Lambda^{*}(\rho)$ if and only if $f(z)$ satisfies (1.4) for some function $g(z)$ in $\Sigma^{*}$; and $f(z)$ is in $\Lambda_{1}^{*}(\rho)$ if and only if $f(z)$ is defined by (1.4) with $g(z)$ in $\Sigma^{*}$ but with the further restriction that $g(z)$ be regular and satisfy (1.1) in $\bar{\Delta}$. Clearly $\Lambda_{1}^{*}(\rho) \subset \Lambda^{*}(\rho)$.

It is evident that $\Lambda(\rho) \subset \Lambda^{*}(\rho)$ for each value of $\rho$. We shall show however that $\Lambda(\rho)$ is a proper subset of $\Lambda^{*}(\rho)$ for some values of $\rho$.

Functions in $\Lambda^{*}(\rho)$ are the reciprocals of weakly starlike, regular, univalent functions introduced by Hummel ([3], [4]). It is for this reason that we refer to members of $\Lambda^{*}(\rho)$ as weakly starlike, meromorphic univalent functions. $\Lambda(\rho)$ and $\Lambda^{*}(\rho)$ are particular cases of classes of functions studied by Styer [8].

It is evident from the above that $\Lambda_{1}^{*}(\rho)=\Lambda_{1}(\rho)$, for all $\rho$. Furthermore, for a given $f(z)$ in $\Lambda^{*}(\rho)$ there exists an increasing sequence of numbers $\left\{r_{n}\right\}$ converging to 1 and a corresponding sequence $\left\{f_{n}(z)\right\}$ such that $f_{n}(z)$ is in 
$\Lambda\left(\rho / r_{n}\right)$, for each $n$, and $\lim _{n \rightarrow \infty} f_{n}(z)=f(z)$ in $\Delta$.

2. Relationship between $\Lambda(\rho)$ and $\Lambda^{*}(\rho)$. The work of Styer [8] shows that $\Lambda(\rho)=\Lambda^{*}(\rho)$ if $\rho<2-\sqrt{3}$. We begin by improving this range slightly.

THEOREM 1. If $\rho<(3-2 \sqrt{2})^{1 / 2}$, then $\Lambda(\rho)=\Lambda^{*}(\rho)$.

Proof. Let $f(z)$ be in $\Lambda^{*}(\rho)$ and let

$$
\psi(z)=\psi(z ; \rho)=-\rho z /(z-\rho)(1-\rho z),
$$

then (1.4) assumes the form $f(z)=\psi(z) g(z)$ for $g(z)$ in $\Sigma^{*}$.

For $\rho \geqslant 2-\sqrt{3}$, Hummel $[3$, p. 548] has essentially shown that

$$
\operatorname{Re}\left\{\frac{z \psi^{\prime}(z)}{\psi(z)}\right\} \leqslant \frac{\left[\left(1-\rho^{2} r^{2}\right)^{1 / 2}-\left(r^{2}-\rho^{2}\right)^{1 / 2}\right]^{2}}{2\left(1-r^{2}\right)\left(1-\rho^{2}\right)}
$$

for $|z|=r$ and $\rho<r<1$. If $g(z)$ is in $\Sigma^{*}$, it is well known [2] that

$$
\operatorname{Re}\left\{\frac{z g^{\prime}(z)}{g(z)}\right\} \leqslant-\left(\frac{1-r}{1+r}\right)
$$

for $|z|=r$. Using the representation for $f(z)$ and the above we get

$$
\begin{aligned}
\operatorname{Re}\left\{\frac{z f^{\prime}(z)}{f(z)}\right\} & =\operatorname{Re}\left\{\frac{z g^{\prime}(z)}{g(z)}\right\}+\operatorname{Re}\left\{\frac{z \psi^{\prime}(z)}{\psi(z)}\right\} \\
& \leqslant \frac{\left(1-\rho^{2}\right)\left(-1+4 r-r^{2}\right)-2\left(1-\rho^{2} r^{2}\right)^{1 / 2}\left(r^{2}-\rho^{2}\right)^{1 / 2}}{2\left(1-r^{2}\right)\left(1-\rho^{2}\right)} .
\end{aligned}
$$

Calculation shows that for $r>\rho>2-\sqrt{3}$, the numerator in (2.4) is negative provided that $(r-1)^{2} Q(r)<0$, where

$$
Q(r)=\left(1+\rho^{2}\right)^{2} r^{2}-\left[6 \rho^{4}-20 \rho^{2}+6\right] r+\left(1+\rho^{2}\right)^{2} .
$$

To insure that $Q(r)$ be negative in some annulus $\rho_{1}<|z|<1$ it is sufficient that $Q(1)<0$; this is the case if $\rho<(3-2 \sqrt{2})^{1 / 2}$.

THEOREM 2. If $\rho>1 / 2$, then $\Lambda(\rho)$ is a proper subset of $\Lambda^{*}(\rho)$.

The proof consists of giving an example and of appealing to the discussion in the introduction. (N. B. $(3-2 \sqrt{2})^{1 / 2} \sim 0.4$; the authors were not able to show the exact relationship between $\Lambda(\rho)$ and $\Lambda^{*}(\rho)$ when $(3-2 \sqrt{2})^{1 / 2} \leqslant$ $\rho \leqslant 1 / 2$.)

For any natural number $k$ and any real number $\theta$ we define

$$
f(z)=-\rho\left(1-e^{i \theta} z^{k}\right)^{2 / k} /(z-\rho)(1-\rho z),
$$


a member of $\Lambda^{*}(\rho)$. Letting $z=e^{i \phi}, 0 \leqslant \phi<2 \pi$, we find that

$$
z f^{\prime}(z) / f(z)=2 i V(\phi)
$$

where

$$
V(\phi)=\frac{-\sin (\theta+k \phi)}{2-2 \cos (\theta+k \phi)}+\frac{\rho \sin \phi}{1-2 \rho \cos \phi+\rho^{2}}
$$

By the conformal properties of (2.6) we conclude that if $V(\phi)$ is nondecreasing, then $f(z)$ is in $\Lambda(\rho)$; on the other hand, if there is an interval over which $V(\phi)$ is decreasing, then $f(z)$ cannot be in $\Lambda(\rho)$. In the discussion which follows we examine the behavior of $V(\phi)$ for arbitrary $k, \theta$ and $\rho$ and then choose appropriate values of these parameters to give a proof of Theorem 2.

Differentiating $V(\phi)$ we find that $V^{\prime}(\phi)=Q(\phi) / P(\phi)$, where $P(\phi) \geqslant 0$ and

$$
\begin{aligned}
Q(\phi)= & (2 k-2 k \cos (\theta+k \phi))\left(1-2 \rho \cos \phi+\rho^{2}\right)^{2} \\
& +\left(\rho \cos \phi-2 \rho^{2}+\rho^{3} \cos \phi\right)(2-2 \cos (\theta+k \phi))^{2} \\
= & 2(1-\cos (\theta+k \phi)) H(\phi),
\end{aligned}
$$

where

$$
\begin{aligned}
H(\phi)= & \left(1-2 \rho \cos \phi+\rho^{2}\right)^{2} k \\
& +\left(\rho \cos \phi-2 \rho^{2}+\rho^{3} \cos \phi\right)(2-2 \cos (\theta+k \phi)) .
\end{aligned}
$$

At this point we can show that $f(z)$ is not in $\Lambda(\rho)$ if $H(\phi)<0$ for some value of $\phi$.

Choose $k=1$ and $\theta=\pi$, then

$$
H(\phi)=(1+\rho)^{2}\left[2 \rho \cos ^{2} \phi-2 \rho \cos \phi+(1-\rho)^{2}\right] .
$$

Replacing $\cos \phi$ by $x,-1 \leqslant x \leqslant 1$, the bracketed expression in (2.10) reduces to the quadratic $B(x)=2 \rho x^{2}-2 \rho x+(1-\rho)^{2} . B(x)$ is strictly decreasing for $-1 \leqslant x<1 / 2$ and strictly increasing for $1 / 2<x \leqslant 1$. $B(1 / 2) \geqslant 0$, if $0<\rho \leqslant 1 / 2$ and $B(1 / 2)<0$, if $1 / 2<\rho<1$; therefore we conclude that $f(z)$ is in $\Lambda(\rho)$ when $0<\rho \leqslant 1 / 2$ and not otherwise.

The following functions

$$
F(z)=-\rho(1+z)^{2} /(z-\rho)(1-\rho z)
$$

and

$$
f(z)=-\rho(1-z)^{2} /(z-\rho)(1-\rho z),
$$


obtained by appropriate choices for $k$ and $\theta$ in (2.5), are useful examples as extremals. As we have already shown $F(z)$ is in $\Lambda(\rho)$ for $\rho \leqslant 1 / 2$ but is in $\Lambda^{*}(\rho) \backslash \Lambda(\rho)$ for $\rho>1 / 2$. As we will now show $f(z)$ is in $\Lambda(\rho)$ for all $\rho$. To this end we choose $k=1$ and $\theta=0$ in (2.9); then

$$
H(\phi)=(1-\rho)^{2}\left[-2 \rho \cos ^{2} \phi-2 \rho \cos \phi+(1+\rho)^{2}\right] .
$$

Replacing $\cos \phi$ by $x$, we obtain

$$
D(x)=-2 \rho x^{2}-2 \rho x+(1+\rho)^{2},
$$

having removed the positive factor $(1-\rho)^{2}$. An examination of the quadratic $D(x)$ reveals that $D(x)$ is positive for all $x$ in the interval $[-1,1]$ and all admissible $\rho$. This is sufficient to ensure that $f(z),(2.12)$, is in $\Lambda(\rho)$ for all $\rho$.

Similar calculations show that when $k=2$ and $\theta=0, f(z)$, defined in (2.5), is in $\Lambda(\rho)$ for all $\rho$; and if $k=2$ and $\theta=\pi$, then $f(z)$ is in $\Lambda(\rho)$ for $0<\rho<[4 \sqrt{2}-\sqrt{5}] / 3 \sqrt{3}$, otherwise $f(z)$ is in $\Lambda^{*}(\rho)$. Also, (2.9) shows that if $k$ is sufficiently large, $f(z)$ is in $\Lambda(\rho)$ for all $\rho$ and any value of $\theta$.

3. Bounds and coefficients. We begin this section by giving bounds on the growth of weakly starlike meromorphic functions and then apply these to determine bounds on their coefficients.

LEMMA 1. If $f(z)$ is in $\Lambda^{*}(\rho)$ and $|z|=r$, then

$$
|f(z)| \leqslant \begin{cases}\rho(1+r)^{2} /(\rho-r)(1-\rho r), & r<\rho, \\ \rho(1+r)^{2} /(r-\rho)(1-\rho r), & r>\rho\end{cases}
$$

and

$$
|f(z)| \geqslant \rho(1-r)^{2} /(r+\rho)(1+\rho r), \quad 0<r<1, \quad r \neq \rho .
$$

These bounds follow from the representation of $f(z)$ in terms of meromorphic starlike functions whose growth bounds are known [2]. Choosing $g(z)=$ $(1+z)^{2} / z$ in (1.4) shows the bounds are sharp.

LEMMA 2. If $f(z)$ is in $\Lambda^{*}(\rho)$ and $f(z)=1+\sum_{n=1}^{\infty} a_{n} z^{n}$ for $|z|<\rho$, then

$$
\left|\rho a_{n}-\left(1+\rho^{2}\right) a_{n-1}+\rho a_{n-2}\right| \leqslant 2 \rho / n, \quad n=1,2,3, \cdots,
$$

and in particular,

$$
(1-\rho)^{2} / \rho \leqslant\left|a_{1}\right| \leqslant(1+\rho)^{2} / \rho \text {. }
$$


PROof. Rewriting (1.4) we get

$$
f(z)(z-\rho)(1-\rho z)-\rho z=g(z),
$$

where $g(z)$ is in $\Sigma^{*}$. If we let $g(z)=1 / z+\Sigma_{k=0}^{\infty} b_{k} z^{k}$ for $z$ in $\Delta$, then it is known [1] that $\left|b_{k}\right| \leqslant 2 /(k+1)$ for all $k$; and this along with some calculation in (3.5) gives (3.3). (3.4) is obtained from (3.3) by choosing $n$ to be 1 .

The function defined by

$$
f_{0}(z)=-\rho(1-z)^{2} /(z-\rho)(1-\rho z)
$$

which is in $\Lambda(\rho)$ shows that (3.3) is sharp for 1 and 2 and that the lower bound of (3.4) is sharp. The functions

$$
f_{n}(z)=-\rho\left(1-e^{i \theta} z^{n}\right)^{2 / n} /(z-\rho)(1-\rho z)
$$

show that (3.3) is sharp for all $n$.

THEOREM 3. If $f(z)$ is in $\Lambda^{*}(\rho)$ and

$$
f(z)=\sum_{n=-\infty}^{\infty} A_{n} z^{n}, \quad \rho<|z|<1 ;
$$

then

$$
\left|A_{-n}\right| \leqslant \rho^{n}((1+\rho) /(1-\rho)), \quad n=1,2, \cdots \cdot
$$

and

$$
\left|A_{n}\right|=O(1 / \sqrt{n})
$$

Proof. For any function $f(z)$ with a simple pole at $\rho$ and otherwise regular in $\Delta$, we may write $f(z)=\rho /(z-\rho) \cdot h(z)$ is regular in $\Delta$. If $h(z)$ $=\Sigma_{k=0}^{\infty} d_{k} z^{k}$, then for $\rho<|z|<1$,

$$
f(z)=\left(\sum_{k=1}^{\infty}\left(\frac{\rho}{z}\right)^{k}\right)\left(\sum_{k=0}^{\infty} d_{k} z^{k}\right)
$$

Comparing coefficients in (3.9) and (3.6) we then have

$$
\begin{aligned}
A_{-n} & =\rho^{n} d_{0}+\rho^{n+1} d_{1}+\rho^{n+2} d_{2}+\cdots \\
& =\rho^{n}\left[d_{0}+d_{1} \rho+d_{2} \rho^{2}+\cdots\right] \\
& =\rho^{n} h(\rho)
\end{aligned}
$$

consequently

$$
\left|A_{-n}\right|=\rho^{n}|h(\rho)|, \quad n=1,2,3, \cdots
$$


If $f(z)$ is now chosen to be in $\Lambda^{*}(\rho)$, then $h(z)=-z g(z) /(1-\rho z)$, with $g(z)$ in $\Sigma^{*}$, the class of normalized meromorphic starlike functions. Hence we may write

$$
|h(\rho)|=\frac{\rho}{\left(1-\rho^{2}\right)}|g(\rho)| \leqslant \frac{\rho}{\left(1-\rho^{2}\right)} \frac{(1+\rho)^{2}}{\rho}=\frac{1+\rho}{1-\rho} .
$$

This with (3.10) gives (3.7).

Next we prove (3.8) by first showing it is true when $f(z)$ is in $\Lambda_{1}(\rho)$ and later remove this restriction. Consequently, let $f(z)$ be in $\Lambda_{1}(\rho)$, then

$$
\frac{-z f^{\prime}(z)}{f(z)} \cdot \frac{(z-\rho)(1-\rho z)}{z}=P(z)
$$

is regular throughout $\bar{\Delta}$ and since the factor $(z-\rho)(1-\rho z) / z$ is positive on $\partial \Delta$ it follows that $\operatorname{Re}\{P(z)\}>0$ for $|z|=1$, therefore $\operatorname{Re}\{P(z)\}>0$ for $z$ in $\bar{\Delta}$. Furthermore if

$$
P(z)=\sum_{n=0}^{\infty} c_{n} z^{n}, \quad z \in \Delta, \quad f(z)=1+\sum_{n=1}^{\infty} a_{n} z^{n} \quad \text { for }|z|<\rho,
$$

then $c_{0}=\rho a_{1}$ and $\operatorname{Re}\left\{\rho a_{1}\right\}>0$.

Using Parseval's identity and the known bounds $\left|c_{n}\right| \leqslant 2\left|c_{0}\right|=2 \rho\left|a_{1}\right|$, $n=1,2,3, \cdots$ (see for example [2]) we have, with $z=r e^{i \theta}$, that

$$
\begin{aligned}
\frac{\mathrm{i}}{2 \pi} \int_{0}^{2 \pi}|P(z)|^{2} d \theta & =\left|c_{0}\right|^{2}+\sum_{k=1}^{\infty}\left|c_{k}\right|^{2} r^{2 k} \\
& \leqslant \rho^{2}\left|a_{1}\right|^{2}+4 \rho^{2}\left|a_{1}\right|^{2} r^{2} /\left(1-r^{2}\right) \\
& =\rho^{2}\left|a_{1}\right|^{2}\left[1+4 r^{2} /\left(1-r^{2}\right)\right] \\
& =\rho^{2}\left|a_{1}\right|^{2}\left[\left(1+3 r^{2}\right) /\left(1-r^{2}\right)\right] .
\end{aligned}
$$

Now restricting $z$ so that $\rho<|z|<1$ and making use of (3.1), (3.11) and the Schwarz inequality we have

$$
\frac{1}{2 \pi} \int_{0}^{2 \pi}\left|z f^{\prime}(z)\right| d \theta \leqslant \frac{\rho r(1+r)^{2}}{(r-\rho)^{2}(1-\rho r)^{2}} \cdot \frac{\rho\left|a_{1}\right|\left(1+3 r^{2}\right)^{1 / 2}}{\left(1-r^{2}\right)^{1 / 2}}
$$

$$
\leqslant \frac{8 \rho^{2}\left|a_{1}\right|}{(1-\rho)^{2}(r-\rho)^{2}\left(1-r^{2}\right)^{1 / 2}} \leqslant \frac{B(\rho)}{(r-\rho)^{2}\left(1-r^{2}\right)^{1 / 2}},
$$

where $B(\rho)=8 \rho^{2}(1-\rho)^{-2}\left(\sup \left|a_{1}\right|\right) \leqslant 8 \rho((1+\rho) /(1-\rho))^{2}$, as was shown above, (3.4). 
From this we obtain

$$
\left|n A_{n}\right| \leqslant \frac{1}{2 \pi r^{n}} \int_{0}^{2 \pi}\left|z f^{\prime}(z)\right| d \theta \leqslant \frac{1}{r^{n}} \frac{B(\rho)}{(r-\rho)^{2}\left(1-r^{2}\right)^{1 / 2}} .
$$

\section{Letting}

$$
h_{n}(r)=r^{n}(r-\rho)^{2}\left(1-r^{2}\right)^{1 / 2},
$$

we now maximize $h_{n}(r)$ for fixed $n$ and $\rho<r<1$. Differentiation of (3.14) gives $h_{n}^{\prime}(r)=r^{n-1}(r-\rho)\left(1-r^{2}\right)^{-1 / 2} q_{n}(r)$ with

$$
q_{n}(r)=-(n+3) r^{3}+(n \rho+\rho) r^{2}+(n+2) r-n \rho .
$$

A calculation shows that $q_{n}(r)$ has a unique root $r_{n}$, $\rho<r_{n}<1$, such that $h_{n}\left(r_{n}\right)$ maximizes $h_{n}(r)$ over the interval $(\rho, 1)$.

Summarizing these results we have

$$
n\left|A_{n}\right| \leqslant B(\rho) / r_{n}^{n}\left(r_{n}-\rho\right)^{2}\left(1-r_{n}^{2}\right)^{1 / 2}
$$

for each $n$; we proceed to study the right side of (3.16). Solving the equation $q_{n}\left(r_{n}\right)=0$ for $n$ we have

$$
n=r_{n}^{2} /\left(1-r_{n}^{2}\right)-2 r_{n} /\left(r_{n}-\rho\right) .
$$

Comparing (3.17) for the cases $n$ and $n+1$ shows that $\left\{r_{n}\right\}$ is an increasing sequence which necessarily converges to 1 .

Using (3.17) we write $r_{n}^{n}$ solely in terms of $r_{n}$ and $\rho$, then we conclude that

$$
\lim _{n \rightarrow \infty} r_{n}^{n}=e^{-1 / 2}
$$

Rewriting (3.17) yields the relation

$$
1-r_{n}^{2}=r_{n}^{2}\left(r_{n}-\rho\right) /\left(n\left(r_{n}-\rho\right)+2 r_{n}\right)
$$

and using (3.19) we reassemble (3.16) to appear as

$$
\sqrt{n}\left|A_{n}\right| \leqslant \frac{B(\rho)\left[\left(r_{n}-\rho\right)+2 r_{n}^{r} / n\right]^{1 / 2}}{r_{n}^{n} r_{n}\left(r_{n}-\rho\right)^{5 / 2}} .
$$

Using (3.18) and the fact that $\lim _{n \rightarrow \infty} r_{n}=1$, we see that the right side of (3.20) converges to $B(\rho) e^{1 / 2}(1-\rho)^{-2}$.

It therefore follows that there is a constant $C(\rho)$, independent of $f(z)$, such that

$$
\sqrt{n}\left|A_{n}\right| \leqslant C(\dot{\rho})
$$


for all $n$ and all $f(z)$ in $\Lambda_{1}(\rho)$.

Suppose $f(z)$ is in $\Lambda(\rho)$, then $f(t z)$ is in $\Lambda_{1}(\rho / t)$ for $\rho<t<1$. Using this together with the fact that $(z-\rho / t)(1-\rho z / t) /(z-\rho)(1-\rho z)$ is real and positive when $|z|=1$, we conclude that

$$
F_{t}(z)=\frac{t(z-\rho / t)(1-\rho z / t)}{(z-\rho)(1-\rho z)} f(t z)
$$

is in $\Lambda_{1}(\rho)$. Letting $F_{t}(z)=\sum_{n=-\infty}^{\infty} B_{n}(t) z^{n}$ for $\rho<|z|<1$, we may rewrite (3.21) to read

$$
\sqrt{n}\left|B_{n}(t)\right| \leqslant C(\rho)
$$

for all admissible $t$. Now as $t$ approaches $1, F_{t}(z)$ approaches $f(z)$ and $B_{n}(t)$ approaches $A_{n}$; this gives the bound (3.21) for all $f(z)$ in $\Lambda(\rho)$. This concludes the proof of (3.8) for $\Lambda(\rho)$.

The proof is easily extended to the class $\Lambda^{*}(\rho)$. If $f(z)$ is in $\Lambda^{*}(\rho)$ it has the form (1.4) and

$$
f_{t}(z)=-\rho z(\operatorname{tg}(t z)) /(z-\rho)(1-\rho z)
$$

is in $\Lambda_{1}^{*}(\rho)$ which is $\Lambda_{1}(\rho)$. Letting $f_{t}(z)=\Sigma_{n=-\infty}^{\infty} B_{n}(t) z^{n}$ we see that (3.23) holds, and letting $t$ approach 1 enables us to conclude that (3.8) holds for $\Lambda^{*}(\rho)$ as well as $\Lambda(\rho)$.

The bounds in (3.7) are rendered sharp by the function

$$
f(z)=-\rho(1+z)^{2} /(z-\rho)(1-\rho z) .
$$

THEOREM 4. If $f(z)$ is in $\Lambda^{*}(\rho)$, then for $z$ in $\Delta$ and $z \neq \rho$

$$
\frac{(1-|a|)^{2}}{|a|\left(1-|z|^{2}\right)} \leqslant\left|\frac{f^{\prime}(z)}{f(z)}\right| \leqslant \frac{(1+|a|)^{2}}{|a|\left(1-|z|^{2}\right)},
$$

and

$$
\begin{aligned}
\mid 1 / 2\left(1-|z|^{2}\right)^{2}\left(f^{\prime \prime}(z) / f^{\prime}(z)\right)- & \left(\bar{z}\left(1-|z|^{2}\right)+a^{-1}\left(1+|a|^{2}\right)\left(1-|z|^{2}\right)\right) \mid \\
& \leqslant 2\left|f(z) / f^{\prime}(z)\right| \leqslant 2|a|\left(1-|z|^{2}\right) /\left(1-|a|^{2}\right),
\end{aligned}
$$

where

$$
a=(\rho-z) /(1-\rho \bar{z}) .
$$

Proof. If $f(z)$ is in $\Lambda_{1}(\rho)$ and $z_{0} \neq \rho$, let

$$
g(z)=\frac{(z-a)(1-\bar{a} z)}{-z a f\left(z_{0}\right)} f\left(\frac{z+z_{0}}{1+\bar{z}_{0} z}\right),
$$


with $a=\left(\rho-z_{0}\right) /\left(1-\rho \bar{z}_{0}\right)$. Since $\left(z+z_{0}\right) /\left(1+\bar{z}_{0} z\right)$ maps $a$ into $\rho$, $f\left(\left(z+z_{0}\right) /\left(1+\bar{z}_{0} z\right)\right)$ has a simple pole at $a$, therefore $g(z)$ has a simple pole at the origin with its residue there equal to 1 . Differentiating (3.27) logarithmically and restricting $z$ so that $|z|=1$ we have

$$
\frac{z g^{\prime}(z)}{g(z)}=2 i \operatorname{Im}\left(\frac{a}{z-a}\right)+\frac{1-\left|z_{0}\right|^{2}}{\left|1+\bar{z}_{0} z\right|^{2}} \cdot \frac{w f^{\prime}(w)}{f(w)}, \quad w=\frac{z+z_{0}}{1+\bar{z}_{0} z} .
$$

The real part of the last term in (3.28) is negative on $\partial \Delta$, therefore we conclude that $g(z)$ is in $\Sigma^{*}$.

The Laurent expansion of $g(z)$ in $\Delta$ is

$$
g(z)=\frac{1}{z}+\left[\frac{f^{\prime}\left(z_{0}\right)\left(1-\left|z_{0}\right|^{2}\right)}{f\left(z_{0}\right)}-\left(\frac{1+|a|^{2}}{a}\right)\right]
$$

$$
\begin{aligned}
& +\left[\frac{f^{\prime \prime}\left(z_{0}\right)\left(1-\left|z_{0}\right|^{2}\right)^{2}-2 \bar{z}_{0}\left(1-\left|z_{0}\right|^{2}\right) f^{\prime}\left(z_{0}\right)}{2 f\left(z_{0}\right)}\right. \\
& \left.-\frac{\left(1+|a|^{2}\right)\left(1-\left|z_{0}\right|^{2}\right) f^{\prime}\left(z_{0}\right)}{a f\left(z_{0}\right)}+a^{-1} \bar{a}\right] z+\cdots .
\end{aligned}
$$

Using well-known bounds on the coefficients of functions in $\Sigma^{*}([1],[7])$, we write (3.24) and (3.25) for $\Lambda_{1}(\rho)$. (3.24) and (3.25) can be extended to all of $\Lambda(\rho)$ by observing that if $f(z)$ is in $\Lambda(\rho)$, then $f(t z)$ is in $\Lambda_{1}(\rho / t)$ for $t$ sufficiently close to 1 . If $f(z)$ is in $\Lambda^{*}(\rho)$, then, as was noted in the introduction, we can choose a sequence of functions $\left\{f_{n}(z)\right\}$ and a sequence of increasing real numbers $\left\{r_{n}\right\}$ converging to 1 such that $f_{n}(z)$ is in $\Lambda_{1}\left(\rho / r_{n}\right)$ and $f(z)$ is the uniform limit of the sequence $\left\{f_{n}(z)\right\}$ on appropriate subsets of $\Delta$.

The function $F(z)=-\rho(1+z)^{2} /(z-\rho)(1-\rho z)$ gives equality on the right-hand side of (3.24) for $z=-r, r \neq \rho$; and $f(z)=\rho(1-z)^{2} /(z-\rho)(1-\rho z)$ gives the left side of (3.24), when $z=-r, r \neq \rho$.

Ladegast gives a bound for the quotient $f^{\prime \prime}(z) / f^{\prime}(z)$ in the case where $f(z)$ is assumed to be univalent only [5, (17), p. 133]. His relation is in form similar to (3.25), however his bound is unbounded in the vicinity of $\rho$ whereas the right side of (3.25) is not.

\section{REFERENCES}

1. J. Clunie, On meromorphic schlicht functions, J. London Math. Soc. 34 (1959), 215-216. MR 21 \#5737.

2. G. M. Goluzin, Geometric theory of functions of a complex variable, GITTL, Moscow, 1952; English transl., Transl. Math. Monographs, vol. 26, Amer. Math. Soc., Providence, R. I., 1969. MR 15, 112; 40 \#308. 
3. J. Hummel, Extremal properties of weakly starlike p-valent functions, Trans. Amer. Math. Soc. 130 (1968), 544-551. MR 36 \#5332.

4. - The coefficients of starlike functions, Proc. Amer. Math. Soc. 22 (1969), 311-315. MR 40 \#4440.

5. K. Ladegast, Beiträge zur Theorie der schlichten Funktionen, Math. Z. 58 (1953), 115-159. MR 15, 24.

6. P. Montel, Leçons sur les fonctions univalentes ou multivalentes, Gauthier-Villars, Paris, 1933.

7. C. Pommerenke, On starlike and convex functions, J. London Math. Soc. 37 (1962), 209-224. MR 25 \#1279.

8. D. Styer, Multivalent meromorphic weakly starlike functions, Dissertation, University of Maryland, College Park, Md., 1970.

DEPARTMENT OF MATHEMATICS, UNIVERSITY OF DELAWARE, NEWARK, DELAWARE 19711 